\title{
On the disaggregation of climatological means and anomalies
}

\author{
Gerd Bürger* \\ Potsdam Institut für Klimafolgenforschung, Postfach 601203, D-14412 Potsdam, Germany
}

\begin{abstract}
The tool $\mathrm{C} 2 \mathrm{~W}$ (climate-to-weather disaggregator) is introduced which is aimed at disaggregating climatological means and anomalies into realistic weather processes. For the core variables of minimum and maximum temperature and precipitation, a probit normalization is conducted which transforms each quantity into one which is normally distributed with mean 0 and standard deviation 1 In this way, the total spatial and seasonal spectrum of climatological variability is filtered out, leaving a 3-dimensional process of 'normalized weather' The climatology is contained in the set of parameters which define the probit function. In a second step, a first order autoregressive model is fitted to the normalized weather process which is then, by construction, globally applicable for any time of the year. The determination of probit parameters is achieved, roughly, by a parameterization of climate variability in terms of climate mean. By way of Monte-Carlo simulations, a universal map can be defined which transmits, in a 1-1 way, information between the climate mean and the probit function in such a way that the means of the simulated weather converge statistically, if simulated long enough, to the given climate mean. The simulated variability, however, is generally not preserved. Depending on the specific region and time of the year, $\mathrm{C} 2 \mathrm{~W}$ exhibits deviations from observed variability, with errors increasing in extreme climates; for most temperate climates, the simulated variability is comparable to the observed. The disaggregation of climatological means is then extended to include various aggregations, such as monthly or seasonal means, by interpreting them as anomalies from the mean. Besides being a simple and handy weather generator, $\mathrm{C} 2 \mathrm{~W}$ is best applied as a postprocessing scheme for gridded data sets, such as those from General Circulation Models (GCMs) or gridded climate maps. In that way, C2W works as a simple link between GCMs and, for example, dynamic global vegetation models. $\mathrm{C} 2 \mathrm{~W}$ is available as a Fortran program module.
\end{abstract}

KEY WORDS: Disaggregation - Weather generator - Climate change scenarios

\section{INTRODUCTION}

The growing use of General Circulation Models (GCMs) to study the impact of enhanced carbon emissions into the atmosphere has been accompanied by a similarly growing appearance and application of weather generators and disaggregation schemes. This is natural since many ecosystem models require daily weather records to function properly, and the direct 'weather' outputs of GCMs, especially of their hydrological variables, can hardly be called realistic. For example, this study originated from the demand for a simple but sound disaggregation module for coupling

•E-mail: buerger@pik-potsdam.de a dynamic global vegetation model (DGVM) to a GCM. Disaggregation here is always with reference to meteorological quantities such as temperature and precipitation, and denotes the generation of short-term processes that would aggregate to some predescribed long-term value. For example, given some aggregated GCM output which is usually much more reliable, say seasonal sums of precipitation, the module should generate a series of daily precipitation amounts which sum up to the original seasonal total, and which can be fed into the DGVM.

As the name suggests, weather generators are used to create processes, mostly on a daily time scale, which have characteristics similar to those observed in daily weather. As these characteristics depend very much on 
the underlying climate a thorough knowledge of that climate is necessary. Hence, a weather generator can be described as a transformer of climatologies to weather, and as such is a special disaggregation scheme. For example, transforming the climate change pattern of a GCM scenario to realistic weather events, which are not reliably modeled by the GCM itself, needs some kind of weather generation. For modeling studies, most notably in hydrology which requires long daily records, such a tool is a welcome help. The climate parameters which are used to describe the daily statistics are usually associated, more or less, with the first 2 statistical moments of mean and variability. These are, however, an appropriate measure only for normally distributed quantities, and are of little help when it comes to variables such as precipitation or cloudiness. For those, one has to look for other measures.

The nucleus of almost all currently applied weather generators in hydrology is a 2-stage model which, first, generates a binary sequence of dry and wet days and, second, models the crucial variables according to the state of this sequence. The sequence itself is usually modeled as a Boolean Markov chain model, with transition probabilities estimated from observed data (see Gabriel \& Neumann 1962). One of the first applications of the 2-stage approach was by Nicks \& Harp (1980), who modeled daily temperature and solar radiation as an autoregressive process of first order; the corresponding parameters were picked depending on whether it was dry or wet. The weather generator designed by Richardson (1981) has been widely applied. Besides the dry-wet sequence, he modeled the 4 core variables of hydrology: precipitation amount, maximum and minimum temperature, and solar radiation. The rainfall amount is generated from some highly skewed distribution such as exponential or gamma. Racsko et al. (1991) pointed to the tendency of the Markov approach to produce a bias for some important quantities such as the length of dry or wet periods, and they were able to refine the weather generator accordingly.

Richardson's (1981) weather generator, and all its relatives, here uniformly acronymed WGEN, have been extensively used by the hydrology and related scientific communities, and is certainly the most successful such tool so far. But there is still some room for improvement: various parameters which enter WGEN depend on time and space, and so limit the applicability of the model. Furthermore, precipitation, which is the most difficult variable to simulate, serves as a key quantity for the modeling as it is the independent driving force of all other variables. It will become evident in the course of this study that precipitation is not decoupled from the rest. This study proposes solutions of how to model all variables jointly by a single, multivariate process.

A weather generator can be understood as a black box with 2 lines of input and 1 line for each output, depending on which variables make up the weather process: the first input line is a white noise process (usually), and the second is an array of climate parameters: they serve to modulate the white noise input in such a way that the output is a weather process that is as realistic as possible. The white noise process is nothing more than an uncorrelated sequence of random numbers which have some standard distribution, say $\mathrm{N}(0,1)$ (that is, normal with mean 0 and standard deviation 1). The climate modulation essentially works as a rescaling of this series in space and time. Hence, for the calibration of the climate parameters a key issue is the question of normalization.

A related problem is that of dealing with climate change scenarios. How does climate change aftect the parameters of the weather generator (which can only be estimated under current climate)? Under the name of 'downscaling' there is an entire field of research which extensively deals with questions of this kind. Among them are studies which utilize weather types for modeling of daily weather or precipitation, e.g. Bardossy \& Plate (1992) and Wilson et al. (1992), both with a different definition of weather regime. The Classification and Regression Tree (CART) scheme was applied by Hughes et al. (1993) to distinguish between dry and wet periods. The CART scheme and the socalled 'analog' method were applied by Zorita et al. (1995) for the design of rainfall generators. Neural nets, which have been successfully applied to a whole range of nonlinear phenomena, might also prove useful for the downscaling of moisture quantities; some results are discussed in Elsner \& Tsonis (1992). Another branch of weather generating activities exists under the BAHC (Biological Aspects of the Hydrological Cycle) umbrella, especially in connection with Focus 4 which itself is phrased 'the Weather Generator'; see Bass (1994) for a fuller account.

\section{PROBIT NORMALIZATION}

Climatology at a given location provides, by definition, a concise description of the long-term statistical nature of the corresponding weather being measured (or modeled) there. Disregarding spectral characteristics, the most comprehensive mathematical object for this purpose is certainly the complete probability distribution function of the variable $X$ in question. If the distribution is normal this function is characterized by just 2 parameters: mean $\mu_{X}$ and variability (or standard deviation) $\sigma_{x}$. Now it is possible to transform any 
unbounded, continuous random variable $X$ to a variable $Y$ which is $\mathrm{N}(0,1)$. To see this, let the distribution function of $X$ at $x$ be $F(x)$. It is clear that the new random variable $F(X)$ is uniformly $(0,1)$-distributed. The fact that $F$, as well as the cumulative $\mathrm{N}(0,1)$-distribution function $\Phi$. are monotone and invertible allows the definition of the 2 functions

$$
\begin{gathered}
Y=\Psi(X)=\Phi^{-1}(F(X)) \\
X=\Psi^{-1}(Y)=\Phi\left(F^{-1}(Y)\right)
\end{gathered}
$$

They provide a 1-1 mapping between $X$ and the new variate $Y$. If $X$ is unbounded and continuous then $Y$ is $\mathrm{N}(0,1)$. The map $\Psi$ is often referred to as the 'probit' (= probability integral transformation) of $X$. The reader should not confuse the probit, as defined by Eq. (1), with the much simpler function of the inverse cumulative normal distribution (i.e. $\Phi^{-1}$ ) which some textbooks unfortunately keep under the same name. For more on probits, see e.g. Ledermann (1984).

The characterization of a given distribution is therefore complete if the corresponding probit function is known. A crucial problem is, hence, to find an appropriate fit to an empirically given probit $\Psi$ (stemming from the empirical distribution function $F$ ). For normal quantities this comes down, as we have seen, to estimating $\mu_{X}$ and $\sigma_{X}$. Using the parameters $\alpha=-\mu_{X} / \sigma_{X}$ and $\beta=1 / \sigma_{X}, \Psi$ is the linear function

$$
Y=\alpha+\beta X
$$

This transformation renders satisfactory normalizations for quantities like air and vapor pressure or temperature, for example, but fails to work for many other, mainly hydrological, quantities such as cloudiness, humidity and, most of all, precipitation. From now on, let $X$ denote either TMN, TMX, or PRC, that is, daily minimum and maximum temperature, and precipitation, respectively; these variables represent, for our purposes, the weather process. For $X=\mathrm{PRC}$, it is obvious that because of the boundary 0 (no negative rain) the corresponding $Y$ cannot be $N(0,1)$ : no value of $Y$ can be smaller than $y_{0}=\Psi(0)$. But one can still transform PRC to a $N(0,1)$-variate by using a simple trick: Instead of defining a function $X \rightarrow Y$ between 2 random variables, one defines a function between individual realizations of $X$ and $Y$, and in fact, this is all that is needed. For each occurrence of $X=0$, numbers $y$ are drawn, from an ensemble of $\mathrm{N}(0,1)$ deviates, until one of them happens to satisfy $y \leq y_{0}$. This is the image of that specific realization of $X=0$. In other words, synthetic values are intraduced for the image of the bound. The result will be a $\mathrm{N}(0,1)$-distributed variable in the whole range between $-\infty$ and $+\infty$,

It is, of course, possible to fit a smooth function to any empirically given probit, but usually not without paying the price of a large number of function parameters (and a loss of significance). Bürger (1996), for example, used B-splines for the fitting. To avoid boundary effects one should keep the number of parameters as small as possible. This is particularly important for precipitation. The sensitivity to boundary effects strongly affects extreme events, and can therefore be responsible for artificial events of heavy rainfall or long dry spells. In this study, the following probit approximation is used for precipitation:

$$
Y=\alpha+\ln \left(X+e^{\beta}\right)
$$

This equation plays a similar role as the brokenpower law in e.g. Bardossy \& Plate (1992). In that study, daily precipitation is transformed to an approximately normal variate using a power function with a broken exponent as the only parameter. The reader who might be puzzled by the choice of the particular function in Eq. (3) is referred to later sections where the drawbacks of relying on just a single parameter are discussed.

To represent the annual cycle appropriately, Eq. (3) is applied independently for each month, that is, one uses in Eq. (1) the monthly cumulative distribution functions $F$. In this way, the global climatology for TMN, TMX, and PRC was reduced to a set of 2 monthly parameters for each variable. The use of 2 characteristic climate parameters compares to many other approaches seen in the literature, for example, Hutchinson et al. (1993) or Richardson (1981). A more probit-like approach is the one by Bardossy \& Plate (1992). Additionally to the broken-power parameter, there is a second parameter, which describes the mean of the normalized series or, equivalently, the threshold for the normalized series beyond which rainfall occurs. The normalized series itself is modeled by an autoregressive process of first order.

Since the PRC distribution is skewed towards small values, many authors prefer to split PRC into a binary series for rainfall occurrence and another series for the PRC amount on a rainy day. For example, WGEN uses a simple, 1-parameter exponential function for the rainfall amount distribution. The binary process is governed by a 2-parameter Markov process. Note that since one of the Markov parameters determines the temporal evolution of the modeled process, the number of pure (nonspectral) climate parameters is again 2 .

For 6 stations with daily observations of TMN, TMX, and PRC between 1 January 1961 and 31 December 1990, each from a typical climate zone (Table 1), Fig. 1 depicts the least-squares fits in Eqs. (2) \& (3) for the TMX and PRC parameters, respectively, for the month of January; TMN behaves very much like TMX. For all 6 stations, the 2-parameter regressions are quite satisfactory, with rms errors varying about 0.2 for TMX and about 0.1 for PRC. This is supported by repeating the same analysis for a set of 994 sites, distributed world- 

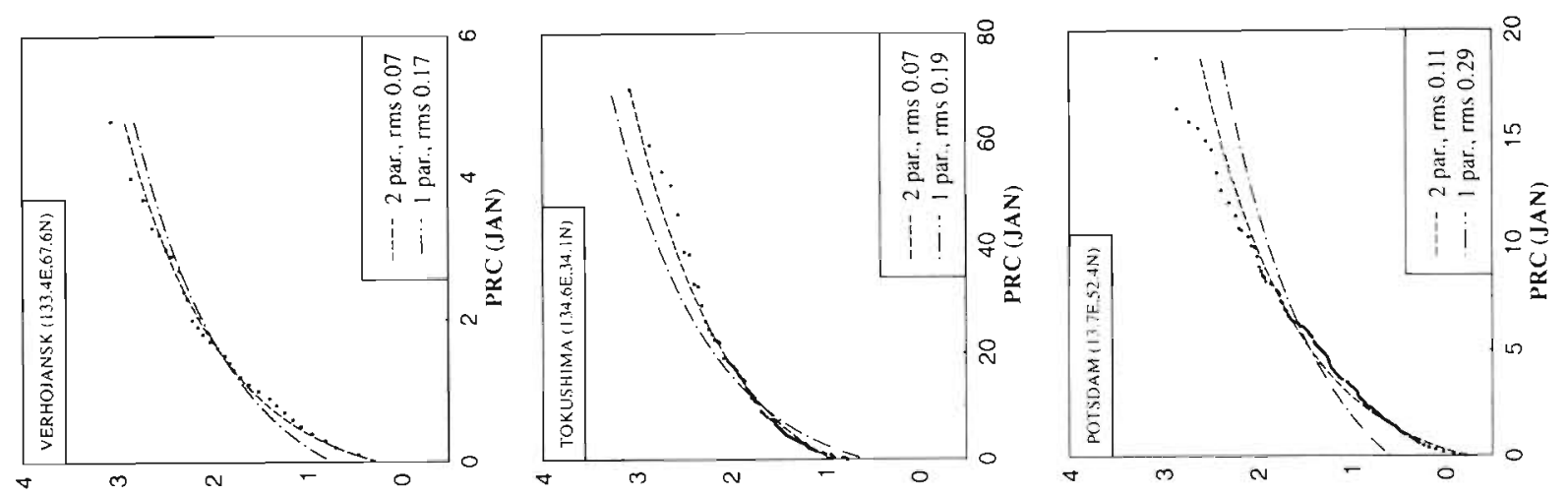

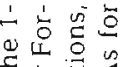

要贾

들

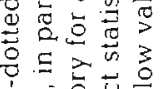

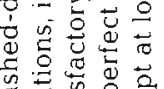

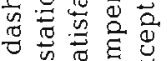

ฮ

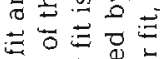

可岀

ه :
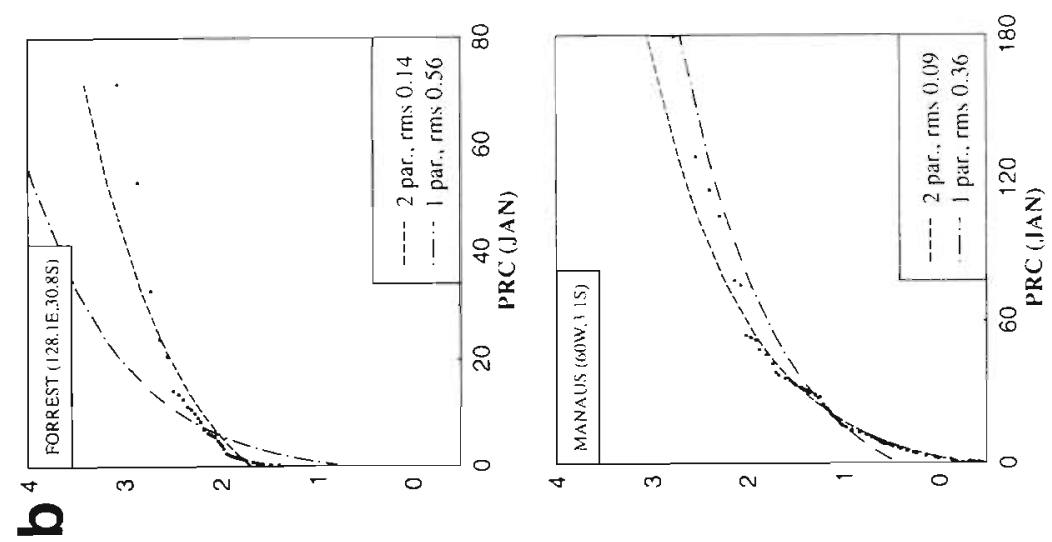

ฮี

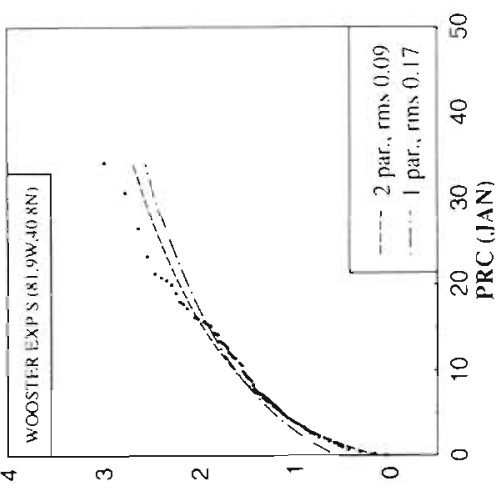

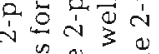

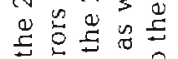

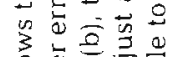

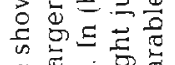

和

을 뭉

空过

$\circ \div$

约学

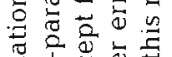

is

象

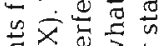

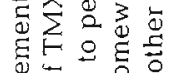
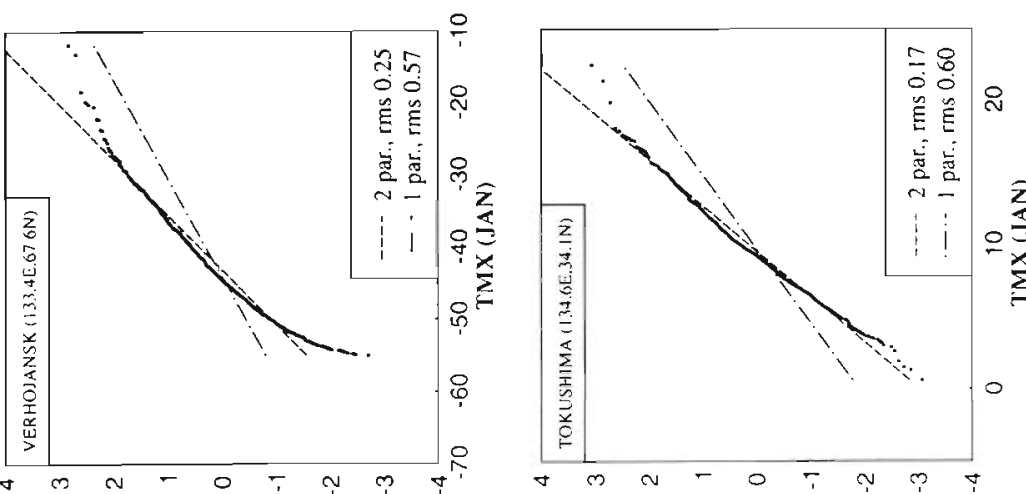

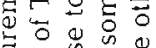

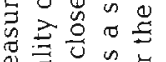

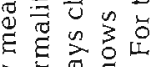

궁

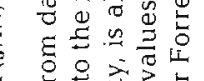

웡

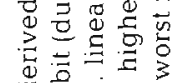

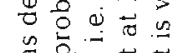

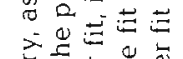

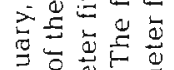

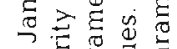

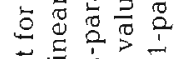
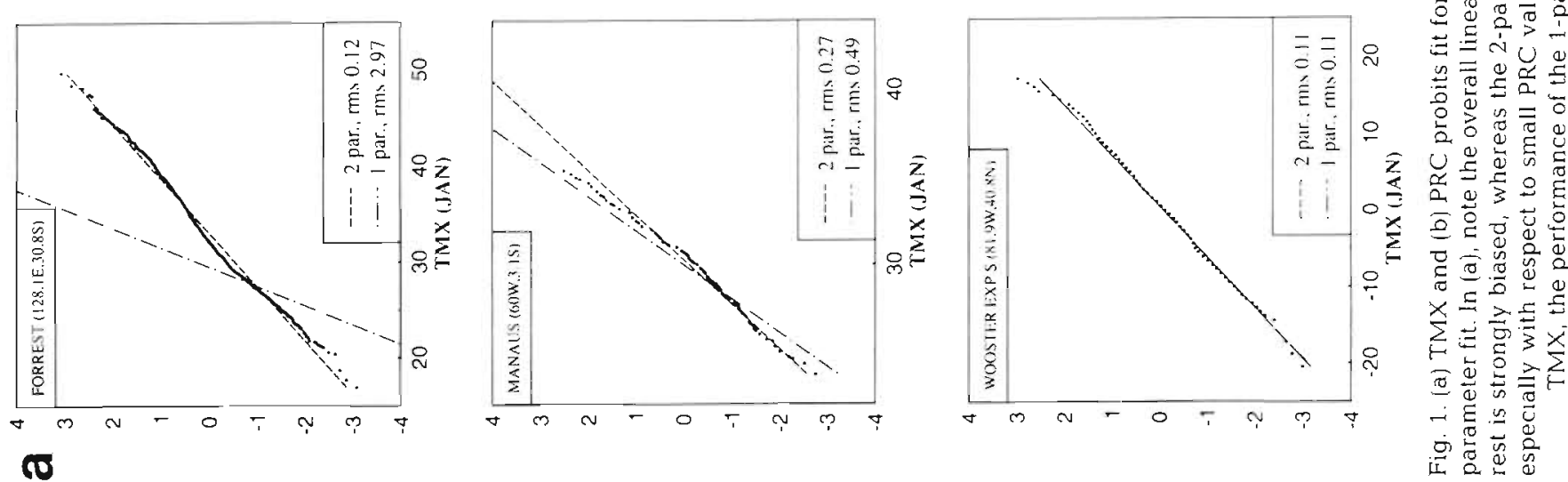
Table 1. The 6 test stations taken from various climatic regions

\begin{tabular}{|lcll|}
\hline Station & Location & Climate & Country \\
\hline Forrest & $128.1^{\circ} \mathrm{E}, 30.8^{\circ} \mathrm{S}$ & Desert & Australı \\
Verhojansk & $133.4^{\circ} \mathrm{E}, 67.6^{\circ} \mathrm{N}$ & Taiga & Russia \\
Manaus & $60^{\circ} \mathrm{W}, 3.1^{\circ} \mathrm{S}$ & Tropical rainforest & Brazil \\
Tokushima & $134.6^{\circ} \mathrm{E}, 34.1^{\circ} \mathrm{N}$ & Wet coastal & Japan \\
Wooster & $81.9^{\circ} \mathrm{W}, 40.8^{\circ} \mathrm{N}$ & Temperate & USA \\
Potsdam & $13.7^{\circ} \mathrm{E}, 52.4^{\circ} \mathrm{N}$ & Temperate & Germany \\
& & & \\
\hline
\end{tabular}

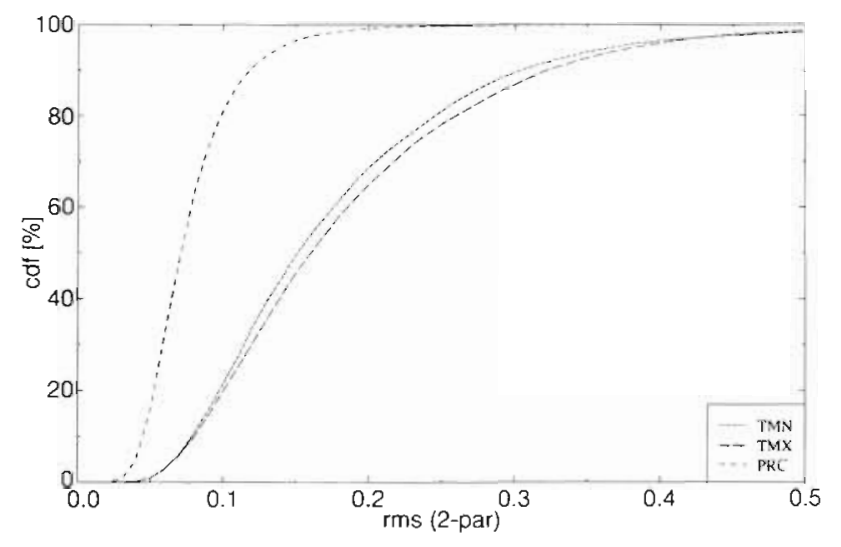

Fig. 2. Cumulative distribution function (cdf) of rms errors for the 2-parameter probit fit for 994 stations with daily measurements (and 12 months) distributed worldwide. For temperature, $60 \%$ of all stations (and months) have errors smaller than 0.2 . For PRC, this rate approaches $100 \%$, and is still $80 \%$ for an rms smaller than 0.1 . The larger errors for temperature indicate that perhaps a non-linear probit approach is necessary

wide except for Africa; again the fit being done independently for each month. The result is shown in Fig. 2. We see that for PRC, more than $80 \%$ of all stations (and months) have an rms smaller than 0.1 ; note, however, that because of the strong non-linearity, small errors in the fit can amplify to significant levels, especially for extreme values. For temperature, this is not the case. The fit errors are, nevertheless, much larger here, which is probably due to the overall assumed normality (i.e. linearity of the probit). In observing that the empirically given probit is sometimes not a smooth curve and that this is very likely due to sampling errors, one can conclude that both fitting functions are correct to a high degree.

\section{CHARACTERIZING GLOBAL CLIMATOLOGIES USING A SINGLE PARAMETER}

It is clear that for each $X$, the pair $(\alpha, \beta)$ depends on the location of the station record. A collection of different climates, therefore, will be represented as a scatter of points $(\alpha, \beta)$ in $\Re^{2}$ (the real plane). The $12 \times 994$ points are shown in Fig. 3 for all 3 variables. To locate a given site in the parameter space of $\alpha$ and $\beta$ we observe the following: Whereas it is clear from Fig. 3 that for an exact description one needs 2 parameters, it is likewise obvious that there exists a strong dependence between both parameters for all $X$. For temperature, translating $\alpha$ and $\beta$ back to $\mu_{X}$ and $\sigma_{X}$, this means that warmer climates are strongly associated with reduced daily variations, a fact which is easily explained by the enhanced heatdamping effect of humidity in warmer climates (tropics). For PRC, we also observe a decreasing $\beta$ with increasing $\alpha$. The interpretation of this dependence is not so obvious since the probit, Eq. (3), is rather complex. However, one can say that variation of the pair $(\alpha, \beta)$ merely creates translations of the probit-graph in the plane $\mathfrak{R}^{2}$, leaving intact the form and (nonlinear) relation between small and large PRC events. Only the offset, and thus the probability per PRC amount, is modified. One can say that the correlation simply reflects the fact that greater PRC probability goes along with greater PRC amounts. This is not unreasonable since the analysis is confined to single months, where variability in the duration of long dry spells, for example, is limited.

For reasons that will become clear in Section 5, a linear regression is now performed of $\beta$ on $\alpha$, for all variables, the results of which are depicted in Fig. 3. One obtains very high correlations of -0.93 and -0.89 for TMN and TMX, respectively. For PRC, the correlation is somewhat less with -0.76 . With the help of the linear functions

$$
\beta=\eta_{x}+\xi_{x} \alpha
$$

one can describe the total $(\alpha, \beta)$ space for each month, and hence the global climatology, by a single parameter. Note that Eq. (4) expresses the same relation on a daily basis that has been described elsewhere for annual values; see e.g. Hutchinson (1995). By feeding this fixed relation in Eq. (4) back into the normalization procedures in Eqs. (2) \& (3), one can formulate a 1parameter probit approach in the following manner:

$$
\begin{aligned}
& Y=\alpha_{T M N}+\left(\eta_{T M N}+\xi_{T M N} \alpha_{T M N}\right) X \\
& Y=\alpha_{T M X}+\left(\eta_{T M X}+\xi_{T M X} \alpha_{T M X}\right) X \\
& Y=\alpha_{\text {PRC }}+\ln \left(X+e^{\left.\eta_{\text {PRC }}+\xi_{\text {PRC }} \alpha_{\text {PRC }}\right)}\right. \\
& X=\left(Y-\alpha_{T M N}\right) /\left(\eta_{T M N}+\xi_{T M N} \alpha_{T M N}\right) \\
& X=\left(Y-\alpha_{T M X}\right) /\left(\eta_{T M X}+\xi_{T M X} \alpha_{T M X}\right) \\
& X=e^{Y-\alpha_{\text {PAC }}}-e^{\eta_{\text {PRC }}+\xi_{\text {PRC }} \alpha_{\text {PRC }}}
\end{aligned}
$$

From the above it can be concluded that, for a given location and month, determination of the daily climatology of the variable $X$ now requires, first, the estimation $\Psi$ of the probit (via the empirically given $F$ ) and, 

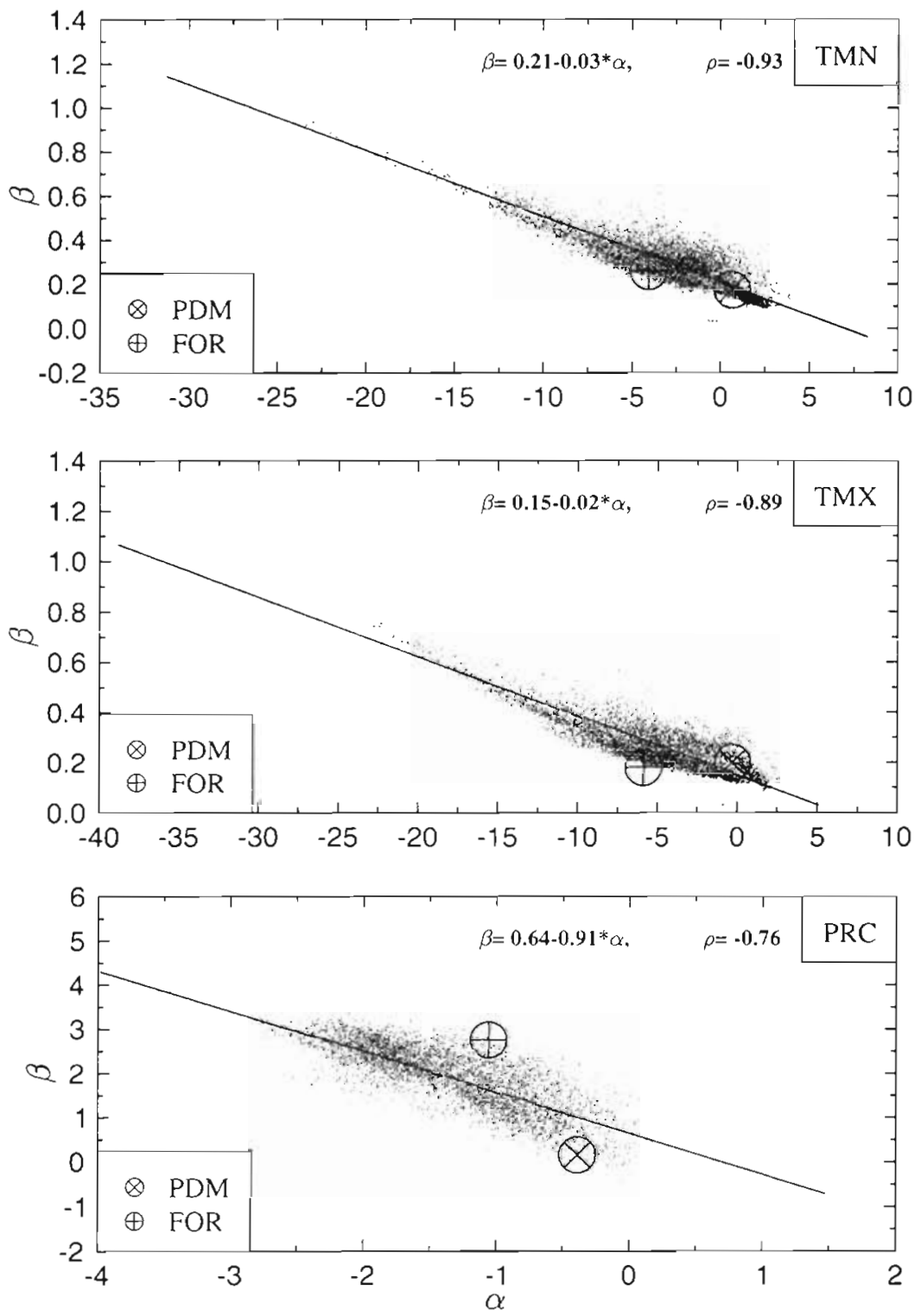

Fig. 3. The scatter of points representing the 2-parameter probit fit $\alpha$ and $\beta$ for the 994 stations and 12 months. For all 3 variables a strong linear relationship is obvious between $\alpha$ and $\beta$. For the temperature values, a dichotomy is visible in the structure of the scatter. There is a tail comprised of more extreme climate separated from the main body of points. Remarkably, both parts reveal almost the same linear dependence between $\alpha$ and $\beta$. The correlation, $\rho$, is higher for the temperature values $(\rho=-0.93$ and $\rho=-0.89$ for TMN and TMX, respectively) than for precipitation $(\rho=-0.76)$. The 2 crosses denote the conditions for Pots$\operatorname{dam}(\otimes)$ and Forrest $(\oplus)$

second, the least-squares calculation of the fit parameter $\alpha_{X}$ using Eq. (5)

The quality loss induced by the parameter reduction is shown in Fig. 4. For the set of 994 stations and the 12 months, Fig. 4 shows the rms errors for both the 2-parameter and 1-parameter fits in a scatter diagram. For the bulk of points the 1-parameter rms error is well below 1 . The performance is best for PRC (but this is very likely due to the non-linear scaling). For TMX, there is a considerable number of stations with the 1-parameter rms error greater than 1, unlike TMN where almost all errors are less than 1 . There are some outliers, mostly stations in extreme climates.

\section{MODELING THE NORMALIZED PROCESS: A WEATHER GENERATOR}

The normalization renders a collection of 994 3-dimensional daily processes $y(t)$ over $30 \mathrm{yr}$ (i.e. of length 10957), the distribution of which is $\mathrm{N}(0,1)$. As a first approximation, we could now generate 'normalized weather' simply by running a random number generator that obeys the covariance between the 3 variables. A better way is to preserve at least some temporal information from the original process $y(t)$. A standard way of doing so is by means of an autoregressive process. The model equation is

$$
y(t+1)=\mathbf{S} y(t)+\varepsilon(t)
$$

with a system matrix $\mathbf{S}$ and a white noise forcing $\varepsilon(t)$ of a certain covariance $\mathbf{Q}$. Denoting the lag-1 and lag-0 covariance $C_{1}$ and $C_{0}$, respectively, the matrices $\mathbf{S}$ and $\mathbf{Q}$ which minimize the expected error $\varepsilon(t)$ in Eq. (6) are calculated as

$$
\begin{aligned}
& \mathbf{S}=\mathbf{C}_{1} \mathbf{C}_{0}^{-1} \\
& \mathbf{Q}=\mathbf{C}_{0}-\mathbf{S C}_{0} \mathbf{S}^{\top}
\end{aligned}
$$

Only here is the importance of using synthetic values for the normalization fully recognized. Without them, Eq. (7) would no longer be applicable. An aiternative, but more complicated estimation of $\mathbf{S}$ and $\mathbf{Q}$ is presented in Bardossy \& Plate (1992). Note that knowledge of both matrices is a pre-

Table 2. Predictive value, in terms of correlations, of the autoregressive model (Eq. 6) without the noise term

\begin{tabular}{|llll|}
\hline & TMN & TMX & PRC \\
\hline lag-1 & $65 \%$ & $74 \%$ & $29 \%$ \\
\hline
\end{tabular}


condition for the combined modeling of all 3 quantities TMN, TMX, and PRC.

According to the probit philosophy, climatic differences are fully contained in the normalization parameters. Consequently, one can assume that the normalized domain has a 'constant climate' and does not require different matrices in Eq. (7). In other words, a globally valid autoregressive model of the 3 normalized variables is assumed. The calculation of Eq. (7) can be carried out jointly for all stations: For example, the lag-1 crosscovariance $\mathbf{C}_{1}$ is calculated by averaging the product $y(t) y(t+1)$ over all instances of 2 consecutive state vectors from all stations. The amount of temporal information retained by the autoregression is shown in Table 2 . The numbers measure the predictive value, in terms of correlations, of a $1 d$ forecast using the deterministic part of Eq. (6), that is, without the noise term. Clearly, the temperature values show a much larger memory compared to precipitation, which always bears strong resemblance to a white noise process.

It is interesting to note that the coupling of temperature and precipitation is not negligible as the sole autocorrelation of PRC is about $24 \%$. Observe that these are global numbers. When defined locally, the corresponding values are, nevertheless, not much better although this very much depends on the considered region. Utilizing a random-number generator with specified input covariance $\mathbf{Q}$ and Eq. (6), one is equipped with a tool to generate a normalized preform of the weather process which is, by construction, universally applicable to the entire globe. Hence, all that is needed for a functioning weather generator is knowledge of the single probit parameter.

Let us, for the rest of this section, turn to the question why we use the 1-parameter normalization instead of the much better 2-parameter approach. In fact, if both parameters were available, the corresponding generator would cover any WGEN-like modeling as well: the
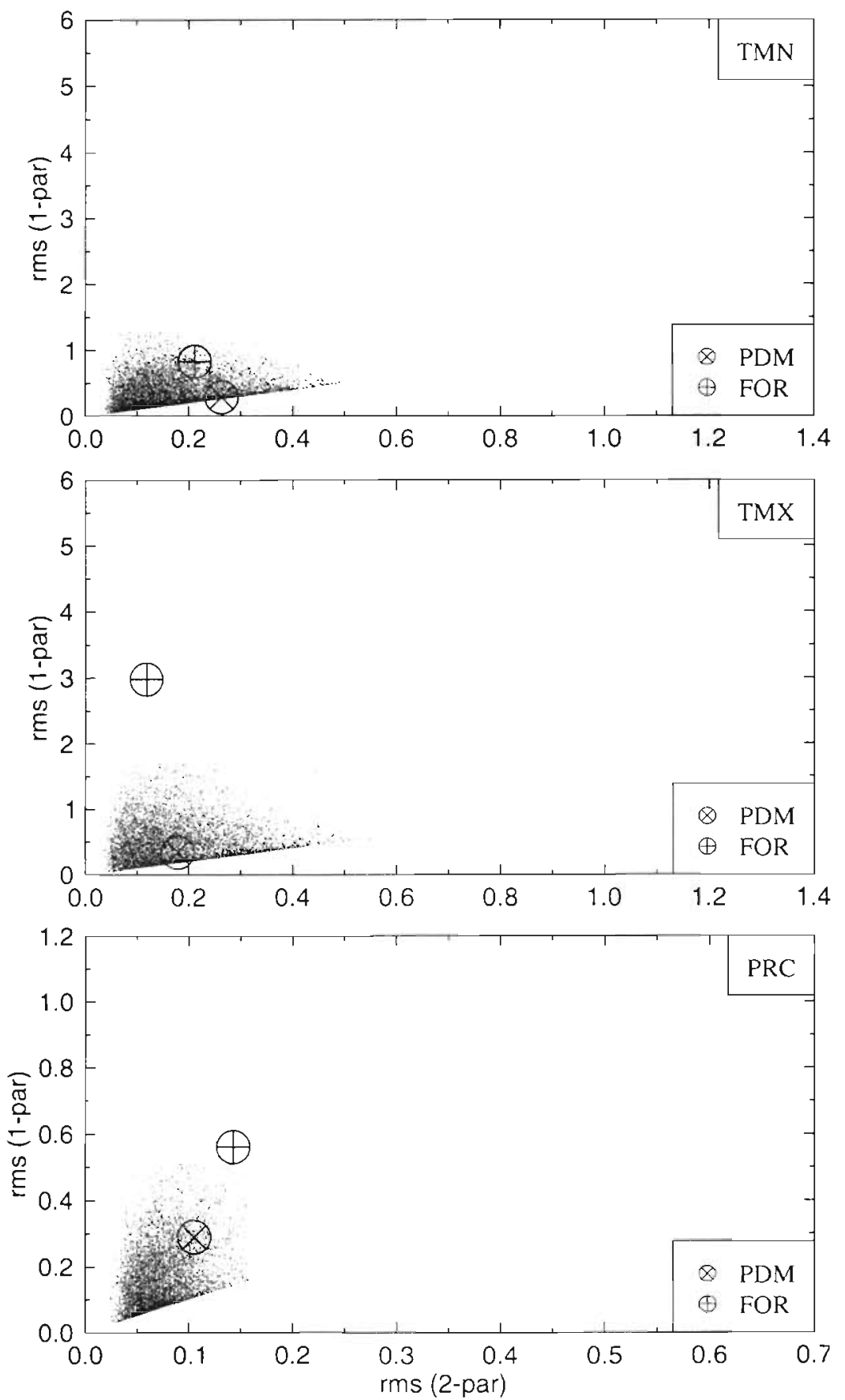

Fig. 4. Comparison, in terms of rms error, between the 2-parameter and the 1 -parameter monthly probit fits. For the 1-parameter fit the error increase is largest for TMX. There are several outliers with errors of between 3 and 5, but the majority of stations still lies well below 1 . A similar situation occurs for PRC. Although most stations have a 1-parameter error of less than 0.4 one sees still quite a few errors reaching 0.8 to 1 . The 2 crosses denote the conditions for Pots$\operatorname{dam}(\otimes)$ and Forrest $(\oplus)$. Note that the 1 -parameter error for Forrest is relatively large for TMX and PRC

Markov chain model for precipitation occurrence can be modeled by the autoregressive method, combined with a threshold value for dry days, and non-linear 
transformations for the amount are inherently modeled via the probit.

The ideal situation for global weather generation is the following: for each month, 2 global, high-resolution maps should exist which describe the first and second probit parameter. Unfortunately, 'exact' estimation of both parameters is limited to those rare locations where long daily weather records are available anyway (and where no weather has to be generated). Independent interpolation of each single parameter between those sites is problematic, in particular for PRC, where the parameters are strongly dependent. One therefore has to rely on currently available databases containing more classical characteristics such as the first few statistical moments of daily weather. Some stations calculate second and third, and sometimes even fourth moments from their records. But with growing moments, again, interpolation becomes problematic. Nevertheless, it is worth trying to find a link between the 2 probit parameters and the 3 or 4 statistical mornents. In short, one should exploit any information that is available. For example, such information might come from monthly statistics. Although the daily characteristics other than the mean appear quite blurred in monthly parameters, there is still a chance of distilling some useful information from them. As a source of such statistics, the program of the Global Historic Climate Network (GHCN; see Eischeid et al. 1995) is a good candidate. It offers, for over 8000 stations worldwide, a whole spectrum of monthly statistics of variables such as TMN, TMX, and PRC. The only complete map of global climatological parameters that is currently available is the map of monthly mean values for the 3 core variables. Here, finally, the 1-parameter probit comes into play. In the following section a method is described of deriving the correct probit parameter $\alpha_{X}$ by just knowing the mean $\mu_{X}$.

\section{ANOMALIES AND THE UNIVERSAL MAP}

It has been mentioned that the weather generator constructed so far is able to produce realistic weather if provided with the appropriate probit parameters. The resulting weather process is then, of course, without any long-term anomalies; anomalies occur merely as a natural (daily) deviation of the random numbers, in the normalized domain, from a zero mean. A systematic deviation from zero, however, will introduce a statistically significant deviation from the climatological mean of the generated weather. In other words, if one adds in Eq. (6) a constant anomaly $\zeta$

$$
y(t+1)=\mathbf{S} y(t)+\varepsilon(t)+\zeta
$$

the resulting denormalized process, i.e. the weather, shows a systematic anomaly $\delta$ from the climatology. Conversely, if one has to normalize weather that shows, say on a monthly scale, an anomaly $\delta$ the resulting normalization will have a non-zero mean $\zeta$. The question is: How are $\zeta$ and $\delta$ related? This is only a trivial problem for normally distributed variables whose probit is linear; here the relation is simply $\zeta=$ $\beta \delta$ (using the $\beta$ from Eq. 2). For non-normal quantities there is no straightforward analytical way to describe the relation.

Summarizing, we can say the following: for each core variable $X$ we are seeking a 2 -dimensional map $\varphi_{X}$

$$
\varphi_{X}=\left(\begin{array}{l}
\mu_{X} \\
\delta_{X}
\end{array}\right) \mapsto\left(\begin{array}{l}
\alpha \\
\zeta
\end{array}\right)
$$

with the following properties: for any long-term climatological mean $\mu_{X}$ and anomaly $\delta_{X}$

- applying the inverse probit $\Psi^{-1}$, i.e. Eq. (5b), with the so found $\alpha$ to a $\mathrm{N}(0,1)$-distributed process by Eq. (6) results in a weather process with expected value $\mu_{X i}$

- if, additionally, the anomaly $\zeta$ is added, cf. Eq. (8), the generated weather exhibits an expected anomaly of $\delta_{X}$.

This turns the weather generator into a disaggregation scheme: Using the map $\varphi_{X}$, one is able to disaggregate climatological and anomaly information of any given time scale into a daily weather process with those characteristics.

Problems of the kind stated above can be solved by means of Monte-Carlo simulations. One starts with a set of $N(0,1)$ distributed variates, denoted by $\mathbf{N}$. Then one chooses an appropriate domain of the dependent parameters, in this case $\alpha$ and $\zeta$, and covers it with a grid of discrete values. Now for each grid point, $(\alpha, \zeta)$,

$$
f(\alpha, \zeta)=\overline{\left\{\Psi_{\alpha}^{-1}(y+\zeta) \mid y \in \mathbf{N}\right\}}
$$

is calculated, that is, the mean of the set $\left\{\Psi_{\alpha}^{-1}(y+\zeta)\right.$ I $y \in \mathbf{N}$ \} with an inverse probit $\Psi_{\alpha}^{-1}$ that uses the parameter $\alpha$ in transformation Eq. (5b). Of course, $\mathbf{N}$ should be large enough so that the mean settles down reliably. If the grid is fine enough, a 2-dimensional surface is defined by $f$ which can be parameterized, for instance, by $B$-splines. In this study, the first variable, $\alpha$, was chosen from the domain $[-40,10]$ for TMX and $[-4,2]$ for PRC, and $\zeta$ varied between $[-5,5]$. The gridding always used 500 sub-intervals. It is clear by construction that the surface is smooth, hence a parameterization is easily possible with arbitrary exactness and without loss in significance. In this study, 2-dimensional B-splines are used with 11 knots in both the $\alpha$ and the $\zeta$ direction. Fig. 5 shows the TMX and PRC surfaces; the TMN surface is not shown as it is very 

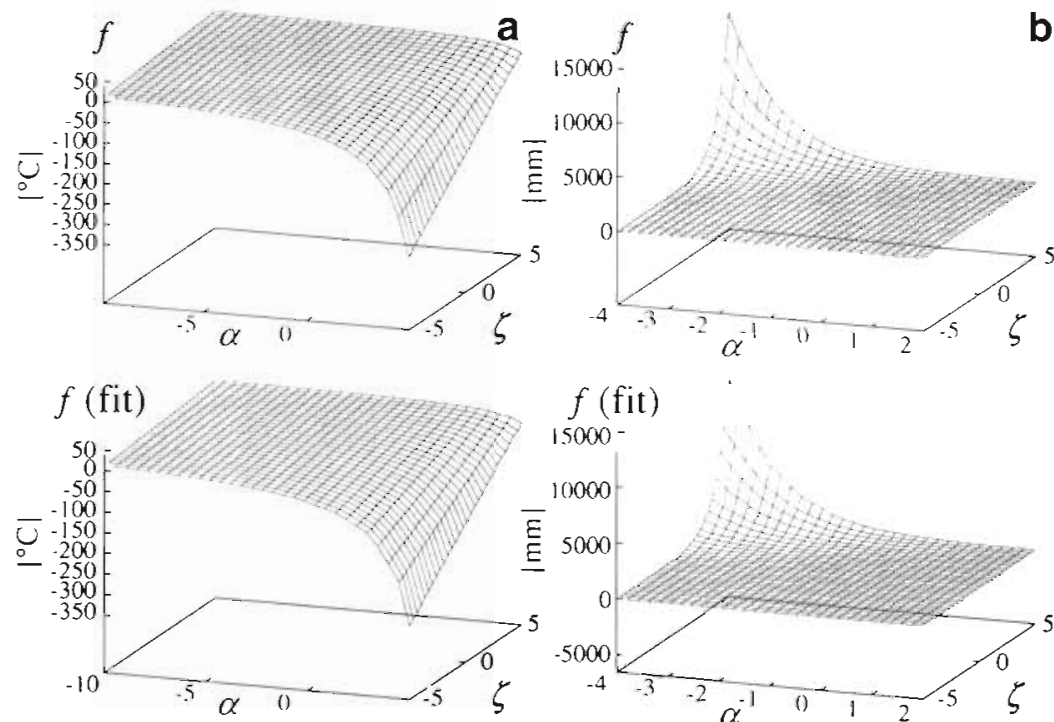

Fig. 5. A section of the 2-dimensional function $f(\alpha, \zeta)$ calculated from the MonteCarlo simulations (top) and fitted by 2-dimensional B-splines (bottom) for (a) TMX and (b) PRC. $f$ describes the expected mean for the weather generator run with probit parameter $\alpha$ and anomaly $\zeta$. Note that the most important part of $f$ is located near the point $(0,0)$ b But in this study, long-term climatology is reflected in the probit parameters while short-term anomalies appear as fluctuations in the normalized space. Also note that, due to the nonlinearities in the normalization, there is a difference between 2 processes which have an identical sum $\mu_{X}+\delta_{X}$ but different $\mu_{X}$ and $\delta_{X}$. Such a difference might even have important consequences with respect to climate change.

The 1-1 link between the climatological parameter $\mu_{X}$ and the parameter $\alpha$ of the weather generator is crucial in this deduction. A second probit parameter, $\beta$, would destroy the balance of the unknowns. In such a case the Monte-Carlo experiments can only determine a 1-dimensional relation between $\alpha$ and $\beta$ and thus leave open 1 degree of freedom. similar to that for TMX. Now consider the 2 functions

$$
\begin{aligned}
& f_{0}(\alpha)=f(\alpha, 0) \\
& g_{\alpha}(\zeta)=f(\alpha, \zeta)
\end{aligned}
$$

As Fig. 5 already suggests, it is clear from Fig. 6 that $f_{0}$ smoothly maps each probit parameter $\alpha$ to some expected value of the corresponding weather process; similarly, it is obvious that this function is monotone and invertible. For the function $g_{\alpha}$, smoothness and invertibility hold correspondingly for any $\alpha$ in question. With the inverse functions, $f_{0}^{-1}$ and $g_{\alpha}^{-1}$, one can define the universal map $\varphi_{X}$ :

$$
\varphi_{X}=\left(\begin{array}{c}
\mu_{X} \\
\delta_{X}
\end{array}\right) \mapsto\left(\begin{array}{c}
\alpha=f_{0}^{-1}\left(\mu_{X}\right) \\
\zeta=g_{\alpha}^{-1}\left(\mu_{X}+\delta_{X}\right)
\end{array}\right)
$$

Having determined, once and for all, the universal map $\varphi_{X}$, with the parameters $\eta_{X}$ and $\xi_{X}$, and the autoregressive matrix $\mathbf{S}$, one is in a position to use the whole scheme as a black box, henceforth denoted as C2W (Climate-toWeather disaggregator). As input, $\mathrm{C} 2 \mathrm{~W}$ takes the 2 variables $\mu_{X}$ and $\delta_{X}$ of long-term climatological mean and some aggregated anomaly, respectively, and returns a weather process of arbitrary length. When simulating long enough, the resulting mean of the process will be exactly $\mu_{X}+\delta_{X}$, that is, the input aggregation.

In this context, the extra splitting into a mean and anomaly term might seem artificial.
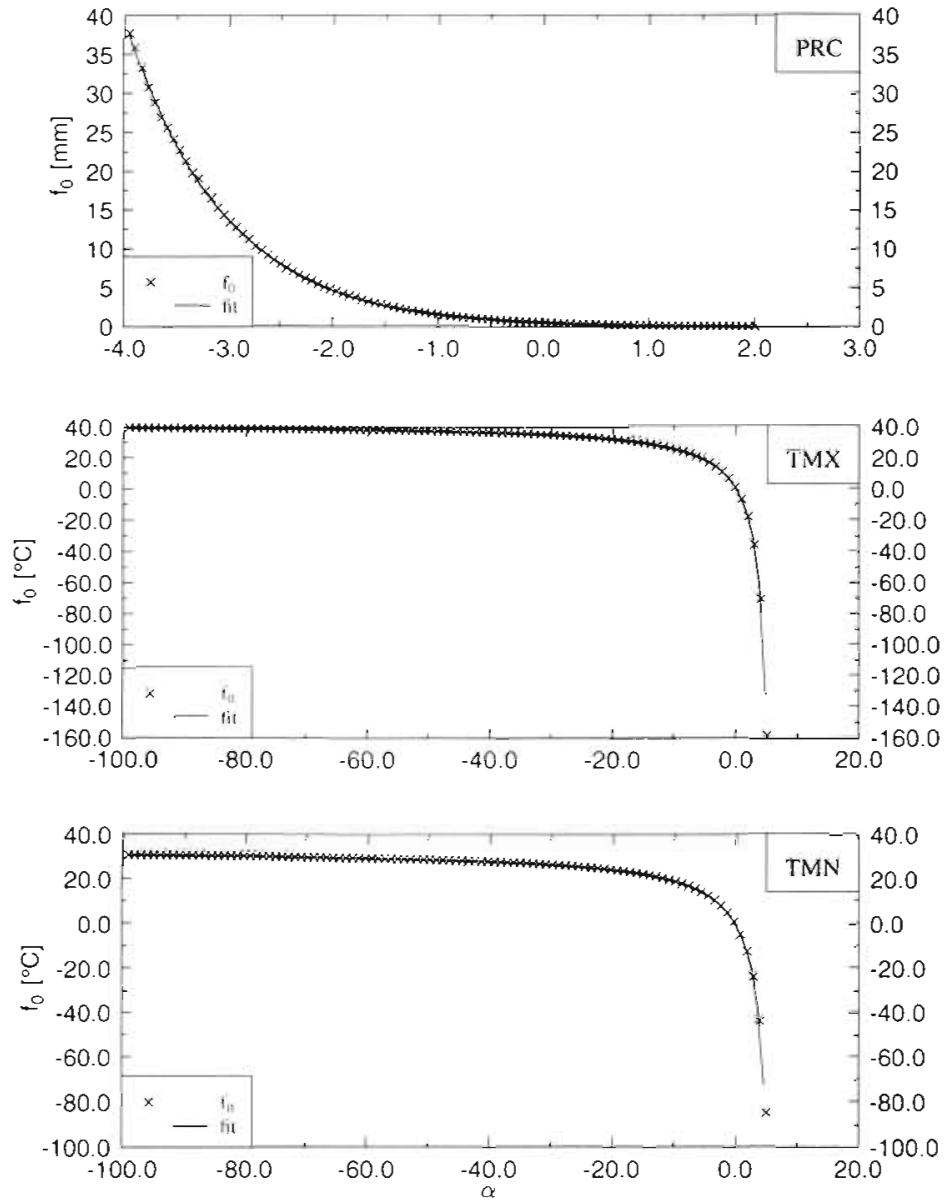

Fig. 6. One-dimensional section $f_{0}(\alpha)=f(\alpha, 0)$ of the function $f, f_{0}(\alpha)$ is monotone and invertible 

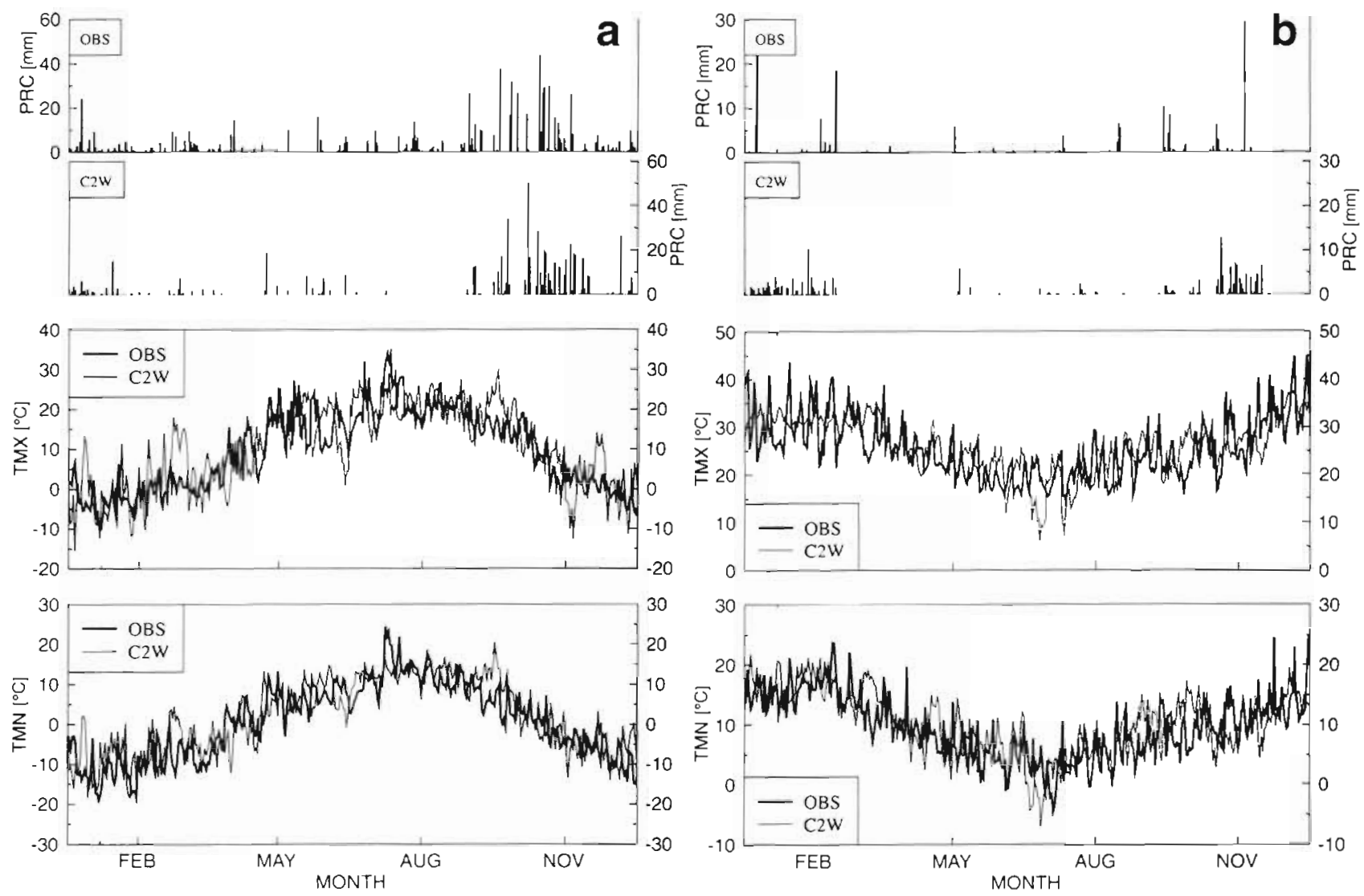

Fig. 7. Comparison between observed weather and disaggregations produced by C2W, for (a) Potsdam, Germany and (b) Forrest, Australia. Note that the observed monthly mean values were fed into $\mathrm{C} 2 \mathrm{~W}$. In (a), climatology and variability are very well reproduced for TMN and TMX. For PRC, there is not quite enough rain throughout the summer, but this is a sampling problem. Note that in fall, the anomalous rainfall amounts are very well reproduced. In (b), compared to Potsdam, the performance is poorer, especially for PRC. Whereas the overall climatology is reproduced the modeling of the summer season is not satisfying. There is a contrast between rare and heavy rainfall events in the observations and frequent and smaller events in the model. This can be understood by referring to Fig. 3 , which shows the relative position of this station far off the regression line

\section{DISAGGREGATING MONTHLY MEANS}

$\mathrm{C} 2 \mathrm{~W}$ is now applied to the record of the station at Potsdam, Germany. First, daily values of TMN, TMX, and PRC were aggregated to long-term monthly means and monthly anomalies relative to those means. These were fed into $\mathrm{C} 2 \mathrm{~W}$ to produce daily values for that period, which were compared with the original observations; a typical section of the outcome is depicted in Fig. 7 Note that autocorrelation and overall variability (i.e. autocovariance) are reproduced quite realistically for both temperature values. Some outliers, such as the too warm period in March, are always possible due to the stochasticity of the model. For PRC, it is obvious that individual rainfall events are not reproduced; but note, for example, that the heavier events in fall are very well matched. There seems to be slightly too little rain throughout the year, but a reversed picture appears in other years, proving the slow settling of PRC statistics.

To test the performance for a much drier climate, the station at Forrest is chosen which lies near the great Victoria desert in southern central Australia. The temperature values again appear realistic, with a possible exception of summer TMX which shows strong variability on a weekly time scale that is not captured by the model. A significant deviation can be seen in the modeled PRC. Although the rainy season in summer is reproduced and the monthly totals are comparable, the modeled PRC distribution is biased towards stronger events. This is very likely due to errors in the linear approach (Eq. 4), as can be seen in Fig. 3.

For a more systematic test of $\mathrm{C} 2 \mathrm{~W}$, a similar test was performed for each of the 994 weather stations. It turned out that after 10 yr of daily values were generated the statistics settled down for all stations and the 
Fig. 8. Grid cell precipitation data taken from the ECHAM1/T21 GCM compared to modeling by $\mathrm{C} 2 \mathrm{~W}$. The GCM weather was aggregated to monthly means and then fed into $\mathrm{C} 2 \mathrm{~W}$. Note the reduced variability in the GCM; this is partly due to the fact that the data represent an entire area (the gridcell), but also due to the limited variability of the GCM with respect to precipitation
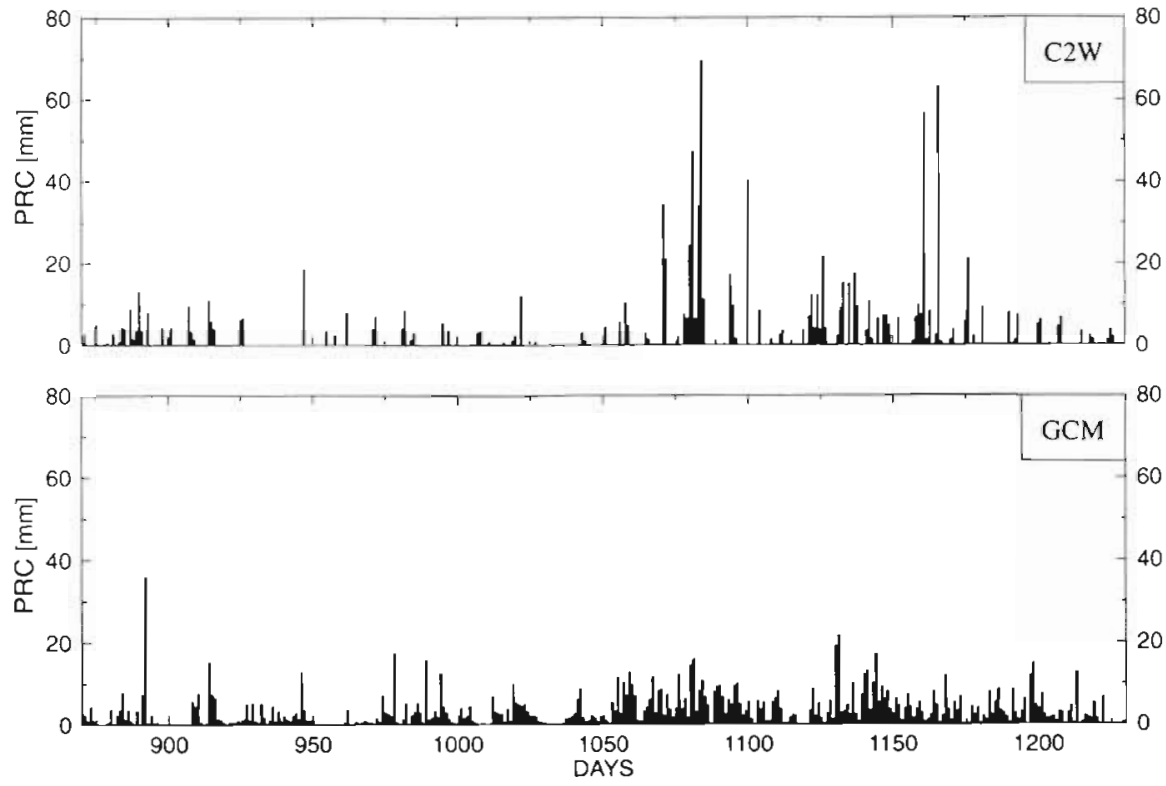

aggregated monthly means for all station values were calculated. The scatter graph of input and output aggregations (not shown) very closely matched the diagonal, that is, the identity function, proving that the estimation of the universal map was correct.

As a final example, a sequence of daily precipitation amounts was taken from an ECHAM1/T21 GCM run (see Roeckner et al. 1992). For a randomly chosen grid cell over Central Europe, the sequence was aggregated to monthly means and the result was fed into C2W. Fig. 8 shows GCM and C2W weather. Note that, since the grid cell represents an entire area of about $500 \mathrm{~km}^{2}$, the GCM series shows much less variability; however, it is well known that the modeling of precipitation is only very poor in practically all GCMs, in particular with respect to their daily variability and their modeling of extreme events. A much more realistic distribution of dry and wet days in the C2W weather can be observed, and a better representation of strong rainfall events. Note that $C 2 \mathrm{~W}$ turns frequent but weak series into a rainfall series of rarer but heavier events.

\section{CONCLUSION}

A new type of climate disaggregation scheme, C2W, is introduced which is meant to be globally applicable. From a given input of long-term climatological means, and possibly some other, e.g. monthly or seasonal, anomalies, it returns a stochastic weather process which shows, when aggregated, exactly those means and anomalies in a statistical way. This means that, while not every such experiment comes out correctly, due to stochastic fluctuations, their expected result does. In the natural climate system, this is quite the same, as any aggregation of meteorological data contains a stochastic element.

To my knowledge, current dynamic vegetation models do not utilize disaggregation schemes other than simple temporal interpolation, such as fitting a sine function to the annual cycle. I also do not know of weather generators that have been applied globally. In this study it is argued that, given this state of the art, $\mathrm{C} 2 \mathrm{~W}$ is an improvement in the following sense. It is shown that by statistically preserving the input aggregation, C2W is, in fact, a disaggregation scheme. The main question is: Is the simulated variability realistic? With respect to the time domain, the modeled equals the observed variability at least to first order, since a corresponding global autoregressive process is applied. The reduction to local autoregressive parameters gives only a minor improvement and introduces a large number of additional parameters. The real difficulty occurs with respect to scaling: Since the scale of day-to-day variability is parameterized by the mean, the spectrum of possible global behavior is considerably reduced by $\mathrm{C} 2 \mathrm{~W}$. This particularly affects more extreme climates where the parameterization is no longer valid. Most temperate climates, however, are modeled relatively well.

An almost perfect global weather generator, for the 3 core variables TMN, TMX, and PRC, could be defined if 2 global, high-resolution maps for each variable, consisting of the first and second probit parameter, were available. Although, for the calibration of C2W, as many as about $1000 \times 12$ pairs of these parameters are 
used, spatial interpolation is not advisable. Because both probit parameters are not independent, considerable errors are likely to be introduced for interpolated pairs. The same argument would hold if the abovementioned parameterization were applied after a 2dimensional probit fitting; but in $\mathrm{C} 2 \mathrm{~W}$, the fitting is done for the 1-parameter probit which came out of the linear regression.

But there is still a need for a high-resolution global map of this particular parameter. At this stage, the corresponding map of mean values enters the picture: Starting from a weather process in the normalized domain, it is obvious that the corresponding mean in the physical domain, that is, after rescaling with the inverse probit, is a function of the probit parameter. Fortunately, this function is invertible, and its inverse defines what I called the universal function: the mapping of any long-term mean to a corresponding weather process that exhibits exactly this mean behavior-in other words, a disaggregation. It is easily extendible to cover anomaly aggregations such as monthly or seasonal means.

Globally gridded mean climatologies have been available for a relatively long time, see e.g. Leemans \& Cramer (1991). C2W can turn them directly into globally gridded weather. The scheme is particularly suited for postprocessing GCM output, which is known to be very imperfect at the daily scale. Given monthly means of the GCM, C2W should improve the 'GCM weather', which can then be fed directly into dynamic global vegetation models. There is probably no better way for validating the proposed scheme than by applying it as an interface between observed global mean climatology and dynamic global vegetation models. A subsequent study of this kind is absolutely necessary.

$\mathrm{C} 2 \mathrm{~W}$ exists as a Fortran program module and can easily be implemented in other numerical schemes. As input it only needs the climatological means and, optionally, anomalies of the 3 core variables; it outputs an appropriate daily weather process of arbitrary length. Extensions to more meteorological variables are under way. Please contact the author for further information or to acquire the module.

Editorial responsibility: Hans von Storch, Geesthacht, Germany

\section{LITERATURE CITED}

Bardossy A, Plate E (1992) Space-time model for daily rainfal using atmospheric circulation patterns. Water Resour Res 28:1247-1259

Bass B (1994) BAHC, Focus 4. Report Nr 4. BAHC Core Project Office, Karlsruhe

Bürger G (1996) Expanded downscaling for generating local weather scenarios. Clim Res 7:111-128

Eischeid JK, Baker CB, Karl TR, Diaz HF (1995) The quality control of long-term climatological data using objective data analysis. I Appl Meteorol 34:2787-2795

Elsner JB, Tsonis AA (1992) Nonlinear predictions, chaos and noise. Bull Am Meteorol Soc 78:49-60

Gabriel R, Neumann J (1962) A Markov chain model for daily rainfall occurrence at Tel Aviv, Israel. Q J R Meteorol Soc 88:99-95

Hughes JP, Lettenmaier DP, Guttorp P (1993) A stochastic approach for assessing the effect of changes in regional circulation patterns on local precipitation. Water Resour Res 29:3303-3315

Hutchinson MF, Richardson CW, Dyke PT (1993) Normalization of rainfall across different time steps. In: National Conference on Irrigation and Drainage Engineering, Park City, Utah, USA. ASCE, p 432-439

Hutchinson MF (1995) Interpolating rainfall using thin plate smoothing splines. Int J Geogr Inf Sys 9:385-403

Ledermann W (ed) (1984) Handbook of applicable mathematics A, Vol VI. Wiley \& Sons, Chichester

Leemans R, Cramer W (1991) The IIASA database for mean monthly values of temperature, precipitation, and cloudiness on a global terrestrial grid. IIASA, Laxenburg

Nicks AD, Harp JF (1980) Stochastic generation of temperature and solar radiation data. J Hydrol 48:1-17

Racsko P, Szeidl L, Semenov M (1991) A serial approach to local stochastic weather models. Ecol Model 57:27-41

Richardson CW (1981) Stochastic simulation of daily precipitation, temperature, and solar radiation. Water Resour Res $17: 182-190$

Roeckner E, Arpe K, Bengtsson L, Brinkop S, Dümenil L, Esch $M$, Kirk E, Lunkeit F, Ponater M, Rockel B, Sausen R, Schleese U, Schubert S, Windelband M (1992) Simulation of the present-day climate with the ECHAM model: impact of model physics and resolution. Report No. 93, Max-Planck-Institut für Meteorologie, Hamburg

Wilson LL, Lettenmaier DP, Skyllingstad E (1992) A multiple stochastic daily precipitation model conditional on largescale atmospheric circulation patterns. J Geophys Res 97. 2791-2809

Zorita E, Hughes JP, Lettemaier DP, von Storch H (1995) Stochastic characterization of regional circulation patterns for climate model diagnosis and estimation of local precipitation. J Clim 8:1023-1042

Manuscript received: January 28, 1997

Revised version accepted: July 21, 1997 